\title{
Angioedema After Local Trauma in a Patient on Angiotensin-Converting Enzyme Inhibitor Therapy
}

\author{
B. Brent Simmons, MD, Michelle A. Folsom, MD, Leslie A. Bryden, MD, and
}

James S. Studdiford, MD, FACP

Angioedema is a side effect that is often associated with the use of angiotensin-converting enzyme (ACE) inhibitor medications. These medications result in increased levels of circulating bradykinins. This case illustrates the result of a local traumatic event to the upper lip, presumably causing marked bradykinin release in a patient who was taking an ACE inhibitor. The local release of bradykinin from trauma, in addition to decreased bradykinin catabolism secondary to ACE inhibitor therapy, resulted in angioedema predominantly in the upper lip. The angioedema resolved with discontinuation of the ACE inhibitor. (J Am Board Fam Med 2008;21:577-9.)

As the indications and usage of angiotensin-converting enzyme (ACE) inhibitor therapy increases, one of its potential side effects, angioedema, is becoming commonly encountered in primary care. Angioedema is a potentially life-threatening side effect of ACE inhibitor therapy, which has an estimated incidence of $0.1 \%$ up to $6 \% .{ }^{1}$ Angioedema resulting secondary to ACE inhibitor use may occur many years after therapy has been initiated. However, it most often occurs within the first week after starting therapy. ${ }^{2} \mathrm{Up}$ to $25 \%$ of reported cases of angioedema seem to be a result of ACE inhibitor therapy. ${ }^{2}$ The other most common causes include allergic reactions, hereditary angioedema, C1 esterase inhibitor deficiency, and idiopathic angioedema. $^{2}$

The mechanism of action of ACE inhibitors includes the blockade of the ACE, which leads to vasodilation. Other actions of ACE inhibitors include blockade of the conversion of substance $\mathrm{P}$ to

This article was externally peer reviewed.

Submitted 20 May 2008; revised 21 July 2008; accepted 29 July 2008.

From Section of Geriatric Medicine, Temple University, Philadelphia, PA (BBS); Calvert Family Practice Associates, Prince Frederick, MD (MAF); Department of Family Medicine, Oregon Health and Science University, Portland (LAB); and the Department of Family and Community Medicine, Thomas Jefferson University, Philadelphia, PA (JSS).

Funding: none.

Conflict of interest: none declared.

Corresponding author: B. Brent Simmons, Temple University Geriatrics, 1316 W. Ontario Street, Philadelphia, PA 19140 (E-mail: b.brent.simmons@tuhs.temple.edu). inactive peptides and the blockade of bradykinin degradation. The increase in circulating substance $\mathrm{P}$ and bradykinin, histamine release, and other mechanisms lead to vasodilatation and increased vascular permeability. ${ }^{1-3}$ Angioedema does not resemble other forms of edema in that it does not appear in dependent areas and is often very transient and localized. ${ }^{4}$ The most important risk factor for developing ACE inhibitor angioedema seems to be African-American race. The mechanism of this increased risk is not clear but may include bradykinin metabolism. Other risk factors include history of angioedema, allergies to seafood, and recent head or neck surgery. ${ }^{2}$

\section{Case}

A 35-year-old African-American woman with a history of hypertension, well controlled (per outpatient chart) with hydrochlorothiazide and lisinopril, presented to the emergency department with a markedly swollen upper lip and a sensation of chest tightness and throat tightness (Figure 1). Lisinopril was started approximately 1 year before this emergency department visit. On the evening before admission, the patient bit her upper lip with moderate force. Shortly thereafter she noted that her lip was markedly swollen. The following morning she saw her primary care physician. Because of continued severe upper lip swelling and persistent feeling of shortness of breath and "throat tightness," she was sent from her primary care physician's office to the emergency department. She denied any recent in- 


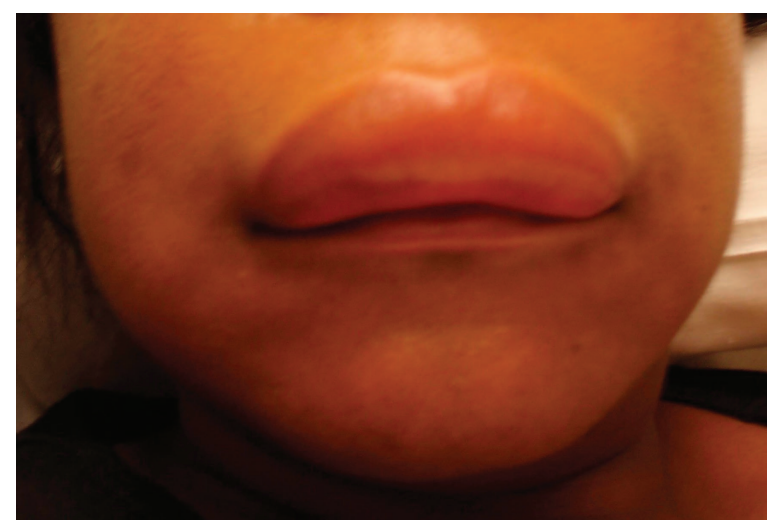

Figure 1. Angiodema confined to the upper lip.

sect bites; consumption of food precipitants (bananas, shrimp, peas, grapes, etc); illicit drug use; herbal medications; and food or drug allergies.

Her medical history was significant only for hypertension. She had no history of angioedema. Medications consisted of hydrochlorothiazide 25 $\mathrm{mg}$ daily, lisinopril $20 \mathrm{mg}$ daily, and an etonogestrel/ethinyl estradiol vaginal ring. There had not been any recent medication changes. Family history was significant for hypertension and diabetes mellitus.

During physical examination her vital signs were temperature, $97.1^{\circ} \mathrm{F}$; pulse ox $97 \%$ on room air; blood pressure 142/99; pulse rate 97; respirations 18; and Body Mass Index score of 40. Her oropharynx was clear and revealed no edema of the tongue or soft palate and there were no tonsillar exudates or ulcerations. There was marked tense edema of the upper lip extending to the lower portion of the nose and obliterating the contour of the nasolabial folds. The lower lip was normal in appearance without edema. Her lungs were clear to auscultation and without wheezing or stridor. She did not have a rash or any other signs of systemic allergic reaction. White blood cell count with differential and sedimentation rate were normal. Urine pregnancy test was negative.

In the emergency department she received epinephrine (1:1000) $0.3 \mathrm{~mL}$ intramuscular once, methylprednisolone $125 \mathrm{mg}$ intravenous once, and diphenhydramine $50 \mathrm{mg}$ intravenous once. She was admitted to the family medicine service for observation and monitoring of her airway, and lisinopril was discontinued. By day 2 of her hospital stay, the angioedema had resolved, her sensation of chest and throat tightness was gone, and she was dis- charged from the hospital on an oral prednisone taper. She was instructed to permanently discontinue all drugs in the ACE inhibitor class and angiotensin receptor blocker class. On the day of discharge, her blood pressure was $128 / 70$ on only hydrochlorothiazide. Further adjustments to her hypertensive medication regimen were deferred to her outpatient follow-up, which was scheduled within 1 week.

\section{Discussion}

The preceding case represents ACE inhibitor-induced angioedema. The patient did not have any allergic symptoms such as urticaria, which makes allergic angioedema unlikely. Other causes of angioedema include hereditary angioedema, pregnancy, lymphoproliferative malignancies, and infection. Given this patient's age of presentation, normal laboratory results, and lack of supportive history or physical findings, these alternate etiologies are very unlikely. Other medications should be considered as a precipitant. However, of this patient's medicine list, lisinopril was the most likely cause and her symptoms resolved after discontinuation whereas hydrochlorothiazide was continued, establishing our diagnosis of ACE inhibitor-induced angioedema.

Multiple case reports have related direct injury caused by perioperative trauma (intubation, oral surgery, anterior dissection of the neck for carotid endarectomy, or cervical laminectomy, etc) as the inciting factor in ACE inhibitor-related angioedema. ${ }^{5-7}$ In this patient the angioedema was confined to the upper lip and its presentation coincided with direct trauma to her upper lip, making trauma a likely precipitant. A MEDLINE search conducted in January 2007 was unable to find another case report describing a specific cause and effect phenomenon related to nonsurgical trauma. The authors hypothesize that the injury caused by biting her upper lip set off a cascade of local events leading to ACE inhibitor-related angioedema.

Bradykinin is a vasoactive peptide causing vasodilatation and increased vascular permeability, and is 10 times more potent than histamine. ${ }^{2,8}$ High levels of circulating bradykinin have been well documented in patients with angioedema. ${ }^{4}$ Although multiple vasoactive peptides have been implicated in angioedema, including substance $\mathrm{P},{ }^{1}$ bradykinin is arguably the most important in ACE inhibitorrelated angioedema. Half of the patients found to 
have ACE inhibitor-related angioedema have a defect in bradykinin metabolism. Similarly, the 4-fold increase in angioedema found in African-American patients is felt to be secondary to genetic differences in the kallikrein-kinin system. ${ }^{4}$

Acute trauma causes activation of bradykinin through the release of factor XII of the clotting cascade. ${ }^{2}$ Bradykinin is then broken down by ACE while passing through the lung. ${ }^{3}$ The blockade of this enzyme by ACE inhibitor leads to both the local and systemic accumulation of bradykinin in susceptible individuals by decreasing its catabolism.

The aforementioned surgical trauma cases resulting in angioedema ascribed this phenomenon to the synergistic effect of increased bradykinin levels. ${ }^{5-7}$ In this patient, the authors postulate that the causative agent was local self-induced trauma to the patient's upper lip. Her lower lip was spared the trauma and hence was entirely free of angioedema. Her shortness of breath during the initial presentation illustrates the systemic nature of angioedema, resulting from higher serum levels of bradykinin.

\section{Conclusion}

This case underscores the fact that everyday trauma such as casually biting one's lip can be enough of a catalyst to cause severe ACE inhibitor-related angioedema in susceptible individuals. This mechanism of accidental self-induced injury is often overlooked in everyday practice. The authors propose that patients on ACE inhibitors should be warned that local trauma has the potential to precipitate severe angioedema. Patients should be advised to report any such instances to their primary care doctor for discussion. Failure to do so could have disastrous consequences, including death from continued administration of the offending agent. This point was emphasized in the recent report of the experience with angioedema in the Antihypertensive and Lipid-Lowering Treatment to Prevent Heart Attack Trial. ${ }^{9}$ However, the ultimate treatment for ACE inhibitor-related angioedema is the same regardless of the cause: permanent discontinuation of all ACE inhibitors. Finally, angiotensin receptor blockers should be used with caution in patients with ACE inhibitor-induced angioedema.
Angiotensin receptor blocker therapy has also been shown to increase bradykinin levels. ${ }^{10}$ Patients who have had ACE inhibitor-induced angioedema are at increased risk to develop angiotensin receptor blocker-induced angioedema; however, the absolute risk is still quite small. ${ }^{11}$ Angiotensin receptor blocker therapy is a reasonable alternative, but the decision to use it should be made by both the patient and the physician after a full discussion of risks and benefits.

\section{References}

1. Byrd JB, Adam A, Brown NJ. Angiotensin-converting enzyme inhibitor-associated angioedema. Immunol Allergy Clin N Am 2006;26:725-37.

2. Vleeming $W$, van Amsterdam JGC, Stricker BHC, deWildt DJ. ACE inhibitor-induced angioedema. Drug Saf 1998;18:171-88.

3. Kaplan AP. Urticaria and angioedema. In: Adkinson, ed. Middleton's allergy: principles and practice, 6th ed. Philadelphia (PA): Mosby; 2003.

4. Agostoni A, Cicardi M. Drug-induced angioedema without urticaria. Drug Saf 2001;24:599-606.

5. Ogbureke KUE, Cruz C, Johnson JV, Helfrick JF. Perioperative angioedema in a patient on long-term angiotensin-converting enzyme (ACE) inhibitor therapy. J Oral Maxillofac Surg 1996;54:917-20.

6. Marrocco-Trischitta MM, Melissano G, de Dominicis D, Chiesa R. Angiotensin-converting enzyme inhibitor-induced angioedema following carotid endarterectomy misdiagnosed as cervical hematoma. Ann Vasc Surg 2006;20:145-7.

7. Krnacik M, Heggeness MH. Severe angioedema causing airway obstruction after anterior cervical surgery. Spine 1997;22:2188-90.

8. Kumar V, Fausto N, Abbas A. Robbins and Cotran Pathologic basis of disease, 7th ed. Philadelphia (PA): Saunders; 2005.

9. Piller LB, Ford CE, Davis BR, et al. Incidence and predictors of angioedema in elderly hypertensive patients at high risk for cardiovascular disease: a report from the Antihypertensive and Lipid-Lowering Treatment to Prevent Heart Attack Trial (ALLHAT). J Clin Hypertens (Greenwich) 2006;8:649_ 56.

10. Cambell DJ, Krum H, Esler MD. Losartan increases bradykinin levels in hypertensive humans. Circulation 2005;111:315-20.

11. Cicardi M, Zingale LC, Bergamaschini L, Agostoni A. Angioedema associated with angiotensin-converting enzyme inhibitor use: outcome after switching to a different treatment. Arch Int Med 2004;164:910-3. 